\title{
POSITRON ANNIHILATION IN $p$-TERPHENYL DOPED WITH BENZOFLUORENE
}

\author{
T. GowOrEK ${ }^{a}$, AND C. RYBKA ${ }^{b}$ \\ ${ }^{a}$ Institute of Physics, Maria Curie-Skłodowska University \\ Pl. M. Curie-Skłodowskiej 1, 20-031 Lublin Poland \\ ${ }^{b}$ Institute of Physics, Technical University of Lublin \\ Nadbystrzycka 38, 20-618 Lublin, Poland
}

\begin{abstract}
In earlier measurements of momentum distribution of annihilating $e^{+} e^{-}$ pairs the intensity of $o$-Ps component seemed to be much higher than that expected from the lifetime data. In this paper we show that such a deviation disappears if one assumes that the pick-off component of angular correlation of annihilation radiation is narrower by $10 \%$ than the component related to free annihilation.

PACS numbers: $78.70 . \mathrm{Bj}, 31.70 . \mathrm{Ks}$
\end{abstract}

\section{Introduction}

In many crystalline organic solids positronium is not formed, but introduction of guest molecules crystallizing together with the main component (host) creates the centers of Ps formation [1]. Momentum distribution of $e^{+} e^{-}$pairs annihilating via two-quantum decay in this case is composed of three parts, related to annihilation of free positrons, para-Ps and pick-off annihilation of ortho-Ps. Decay of para-Ps produces a so-called narrow component; its width is determined by the uncertainty principle. Two remaining parts of momentum distribution are characterized by larger momenta contributed mainly by involved molecular electrons. In principle, their distribution for "free" annihilation and pick-off process needs not be identical. Usually the pick-off component is slightly narrower, e.g. in liquid benzene the free annihilation gives the distribution width $9.57 \times 10^{-3} \mathrm{~m}_{0} \mathrm{c}$ (FWHM) and pick-off annihilation $7.27 \times 10^{-3} m_{0} c$ [2]. However, in our early experiments [1] we found that in the case of doped $p$-terphenyl these two components are of the same shape. On this assumption, well confirmed for larger momenta, full consistency of data about Ps formation intensity was obtained: the intensity of long-lived component $I_{3}$ in the positronium annihilation lifetime spectroscopy (PALS) spectra was 3 times larger than the intensity of narrow component $I_{\mathrm{N}}$ in angular correlation of $2 \gamma$ annihilation. There was one exception from this proportion, $p$-terphenyl doped with benzofluorene, where the $I_{\mathrm{N}}$ value deviated evidently from the lifetime data. This case seems worth a more detailed study. 


\section{Experimental}

Samples in a form of policrystalline pellets were obtained by melting p-terphenyl with an appropriate amount of benzofluorene. Angular correlations of annihilation radiation (ACAR) were determined using a standard long-slit device with resolution $1.1 \mathrm{mrad}$; the total number of collected coincidences was $1.1 \times 10^{6}$ per spectrum. ACAR distribution was measured for a $p$-terphenyl sample containing $0.25 \%$ of benzofluorene. At this level of doping the long-lived component in the lifetime spectrum was $18.3 \%$. The respective lifetime was $1.1 \mathrm{~ns}$, thus $I_{3}$ is practically equal to total $o$-Ps intensity; the share of three-gamma decay can be neglected. As a reference the ACAR for pure $p$-terphenyl was also registered. The lifetime data were taken from Ref. [1].

\section{Results and discussion}

The shape of distribution curves cannot be approximated by single (or two) Gaussians, thus to a void systematic errors no curves were fitted to the experimental

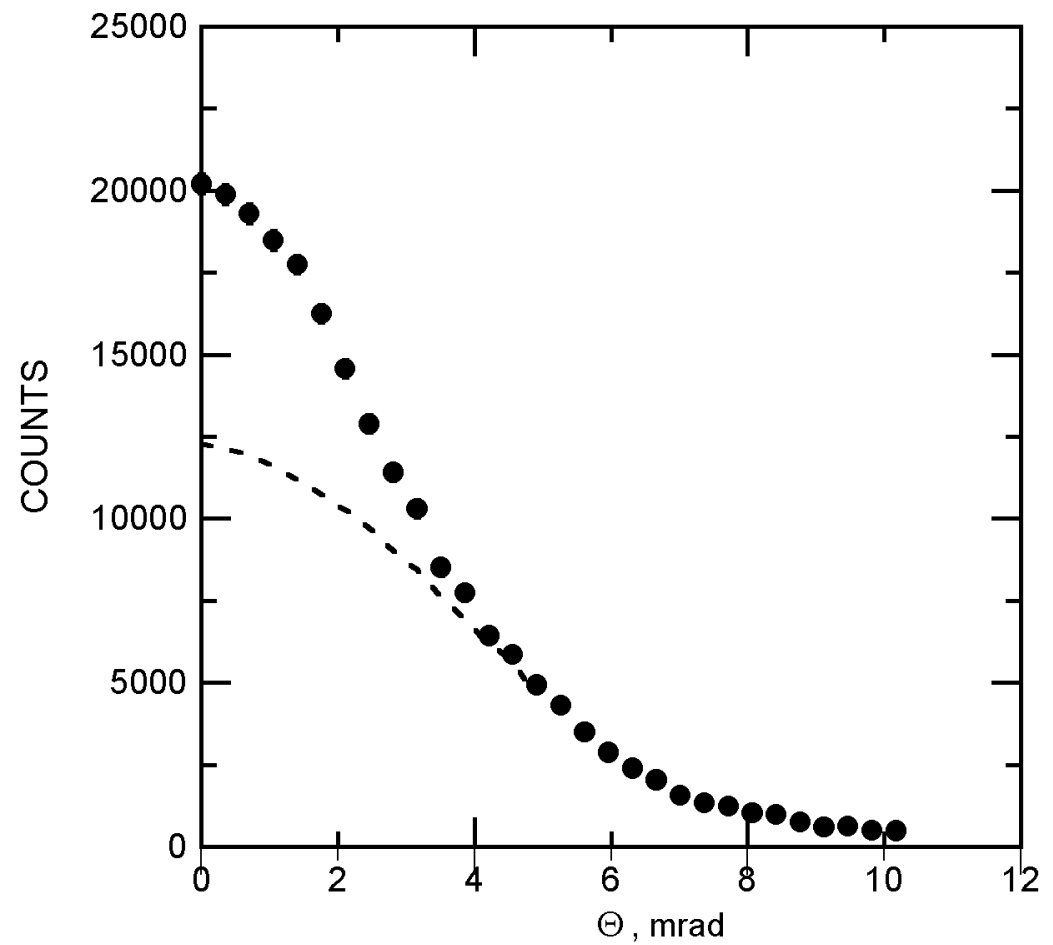

Fig. 1. Momentum distribution of $e^{+} e^{-}$pairs annihilating via positronium formation in $p$-terphenyl doped with benzofluorene (the difference of normalized distributions for doped and pure samples). Dashed curve separates the ortho-from para-part. 
data and all operations were performed on numerical data directly from the experiment. If the long-lived component intensity is $I_{3},\left(1-4 I_{3} / 3\right)$ of total area $S_{d}$ under the ACAR curve belongs to annihilation of free positrons. At the admixture content of 0.25 molar percent its influence on the momentum spectrum is negligible, and the discussed part of the spectrum should be identical with that for pure $p$-terphenyl. Thus, $S_{p}$ area of pure $p$-terphenyl distribution was normalized to $\left(1-4 I_{3} / 3\right) S_{d}$ and subtracted from the distribution for the doped sample. The difference represents thus the momentum distribution for $p$-Ps and pick-off of $o$-Ps. This difference is shown in Fig. 1. The narrow component does not extend farther than $|\theta|=4 \mathrm{mrad}$, thus the region above 4 mrad represents a high momentum fragment of pick-off distribution. It was found that this fragment is not identical with the respective fragment for pure $p$-terphenyl (a larger slope at the same area of fragments). The momentum distribution is narrower than in a pure sample, however, it becomes identical when the angle $\theta$ is scaled down by $10 \%$ (narrowing the distribution by $10 \%$ ). Assuming that this narrowing concerns the whole distribution we extrapolated it down to $\theta=0$ (dashed curve in Fig. 1). The area above the dashed curve represents the momentum distribution for $p$-Ps. The ratio of the areas for $o$-Ps and $p$-Ps is $2.96 \pm 0.04$, thus as expected from the statistical weights of para and ortho states. The width $\Gamma_{\mathrm{N}}$ of narrow component is $4.45 \mathrm{mrad}$, well correlated with the existing data on $\Gamma_{\mathrm{N}}$ and lifetime $\tau_{3}$ for other mixed crystals.

\section{Conclusions}

The anomalous ratio of para and ortho intensities, observed earlier for $p$-terphenyl doped with benzofluorene can be avoided if one assumes that the momentum distribution for pick-off annihilation is narrower by $10 \%$ as compared to that for annihilation of free positrons.

\section{References}

[1] T. Goworek, C. Rybka, J. Wawryszczuk, Phys. Status Solidi B 89, 253 (1978).

[2] W.H. Magalhães, J.-C. Abbé, G. Duplâtre, Struct. Chem. 2, 399 (1991). 